

\title{
Asymptotic representations for Fibonacci reciprocal sums and Euler's formulas for zeta values
}

\author{
par CARsten ELSNER, Shun SHIMOMURA et IEKATA SHIOKAWA
}

RÉSUMÉ. Nous présentons les représentations asymptotiques pour certaines sommes des réciproques des nombres de Fibonacci et des nombres de Lucas quand un paramètre tend vers une valeur critique. Comme cas limite de nos résultats, nous obtenons les formules d'Euler pour les valeurs des fonctions de zeta.

ABSTRACT. We present asymptotic representations for certain reciprocal sums of Fibonacci numbers and of Lucas numbers as a parameter tends to a critical value. As limiting cases of our results, we obtain Euler's formulas for values of zeta functions.

\section{Introduction}

Let $\left\{F_{n}\right\}_{n \geq 0}$ and $\left\{L_{n}\right\}_{n \geq 0}$ be the Fibonacci numbers and the Lucas numbers, defined by

$$
\begin{array}{llll}
F_{0}=0, & F_{1}=1, \quad F_{n+2}=F_{n+1}+F_{n} & (n \geq 0), \\
L_{0}=2, \quad L_{1}=1, \quad L_{n+2}=L_{n+1}+L_{n} & (n \geq 0) .
\end{array}
$$

For various reciprocal sums of these numbers, transcendence and algebraic relations were studied. Duverney, Ke. Nishioka, Ku. Nishioka, and the last named author [3] (see also [2]) proved the transcendence of the numbers $\sum_{n=1}^{\infty} F_{n}^{-2 s}, \sum_{n=1}^{\infty} L_{n}^{-2 s}, \sum_{n=1}^{\infty} F_{2 n-1}^{-s}, \sum_{n=1}^{\infty} L_{2 n}^{-s}(s \in \mathbb{N})$ by using Nesterenko's theorem ([10]) on Ramanujan functions. In [4], for $\zeta_{\mathrm{F}}(s):=$ $\sum_{n=1}^{\infty} F_{n}^{-s}$, we proved that the values $\zeta_{\mathrm{F}}(2), \zeta_{\mathrm{F}}(4), \zeta_{\mathrm{F}}(6)$ are algebraically independent, and that for any integer $s \geq 4$

$$
\zeta_{\mathrm{F}}(2 s)-r_{s} \zeta_{\mathrm{F}}(4) \in \mathbb{Q}\left(\zeta_{\mathrm{F}}(2), \zeta_{\mathrm{F}}(6)\right)
$$

with some $r_{s} \in \mathbb{Q}$; for example

$$
\begin{aligned}
\zeta_{\mathrm{F}}(8)- & \frac{15}{14} \zeta_{\mathrm{F}}(4)=\frac{1}{378(4 u+5)^{2}}\left(256 u^{6}-3456 u^{5}+2880 u^{4}+1792 u^{3} v\right. \\
& \left.-11100 u^{3}+20160 u^{2} v-10125 u^{2}+7560 u v+3136 v^{2}-1050 v\right)
\end{aligned}
$$

with $u:=\zeta_{\mathrm{F}}(2)$, and $v:=\zeta_{\mathrm{F}}(6)$. 
Assume that $\alpha, \beta \in \mathbb{C}$ satisfy

$$
\alpha \beta=-1, \quad|\beta|<1 .
$$

Let $\left\{U_{n}\right\}_{n \geq 0}$ and $\left\{V_{n}\right\}_{n \geq 0}$ be sequences defined by

$$
U_{n}=\frac{\alpha^{n}-\beta^{n}}{\alpha-\beta}, \quad V_{n}=\alpha^{n}+\beta^{n} \quad(n \geq 0),
$$

which are generalized Fibonacci and Lucas numbers, respectively. Indeed, if $\beta=(1-\sqrt{5}) / 2$, then $U_{n}=F_{n}, V_{n}=L_{n}$. For $s \in \mathbb{N}$ consider the reciprocal sums

$$
\begin{aligned}
& h_{2 s}:=(\alpha-\beta)^{-2 s} \sum_{n=1}^{\infty} \frac{1}{U_{2 n}^{2 s}}, \\
& g_{2 s-1}^{*}:=\sum_{n=1}^{\infty} \frac{(-1)^{n+1}}{V_{2 n-1}^{2 s-1}},
\end{aligned}
$$

which are holomorphic for $|\beta|<1$. For $\left\{h_{2 s}\right\}_{s \in \mathbb{N}}$ (respectively, $\left\{g_{2 s-1}^{*}\right\}_{s \in \mathbb{N}}$ ) algebraic relations were discussed in [6] (respectively, [5]). These sums may also be regarded as functions of the modulus $k$ of Jacobian elliptic functions (see Sections 4 and 5, also [4], [5], [6]).

In this paper we present asymptotic representations for these sums as $\beta \rightarrow-1+0$ (or $k \rightarrow 1-0$ ). Degenerate cases of our expressions coincide with Euler's formulas for $\zeta(2 s)=\sum_{n=1}^{\infty} n^{-2 s}$ and $L(2 s-1)=\sum_{n=1}^{\infty}(-1)^{n+1}(2 n-$ $1)^{-(2 s-1)}$, respectively (see Section 3 ).

\section{Statement of results}

Let $B_{2 \nu}$ and $E_{2 \nu}(\nu=0,1,2, \ldots)$ denote, respectively, the Bernoulli and the Euler numbers:

$$
\begin{array}{llll}
B_{0}=1, & B_{2}=1 / 6, & B_{4}=-1 / 30, & B_{6}=1 / 42, \ldots ; \\
E_{0}=1, & E_{2}=-1, & E_{4}=5, & E_{6}=-61, \ldots,
\end{array}
$$

and let $s$ be a positive integer. For $s \geq 2$, let $\sigma_{1}(s), \ldots, \sigma_{s-1}(s)$ be the elementary symmetric functions of the $s-1$ numbers $-1^{2},-2^{2}, \ldots$, $-(s-1)^{2}$ defined by

$$
\sigma_{i}(s)=(-1)^{i} \sum_{1 \leq r_{1}<\ldots<r_{i} \leq s-1} r_{1}^{2} \cdots r_{i}^{2} \quad(1 \leq i \leq s-1),
$$

and for $s \geq 0$, set $\sigma_{0}(s)=1$. For $s \geq 1$ let $\tau_{1}(s), \ldots, \tau_{s}(s)$ be the elementary symmetric functions of the $s$ numbers $-1^{2},-3^{2}, \ldots,-(2 s-1)^{2}$ given by

$$
\tau_{i}(s)=(-1)^{i} \sum_{1 \leq r_{1}<\cdots<r_{i} \leq s}\left(2 r_{1}-1\right)^{2} \cdots\left(2 r_{i}-1\right)^{2} \quad(1 \leq i \leq s),
$$

and for $s \geq 0$ set $\tau_{0}(s)=1$. Our results are given as follows: 
Theorem 1. For $-1<\beta<-1+\delta_{0}$, we have

$$
\begin{gathered}
\left(\alpha^{2}-\beta^{2}\right)^{2 s} h_{2 s}=\Phi_{s}(\eta)\left(1+O\left(e^{-\pi^{2} /(2 \eta)}\right)\right), \\
\eta:=-\log (-\beta)=(1+\beta)(1+O(1+\beta)) .
\end{gathered}
$$

Here $\delta_{0}$ is a sufficiently small positive number, and $\Phi_{s}(X)$ is an entire function written in the form

$$
\Phi_{s}(X):=\frac{2^{s}}{(2 s-1) !}\left(X^{-2}(\cosh (4 X)-1)\right)^{s} \sum_{p=1}^{s} \Lambda_{p}(X)
$$

with

$$
\Lambda_{p}(X):= \begin{cases}\frac{\sigma_{s-1}(s)}{96} X^{2 s-2}\left(\pi^{2}-12 X+4 X^{2}\right), & \text { if } p=1 \\ \frac{(-1)^{p-1}}{2^{2 p+2} p} \sigma_{s-p}(s) B_{2 p} X^{2 s-2 p}\left(\pi^{2 p}-(-1)^{p} 2^{2 p} X^{2 p}\right), & \text { if } p \geq 2 .\end{cases}
$$

Several coefficients of the series expansion

$$
\Phi_{s}(X)=\sum_{j=0}^{\infty} \lambda_{j}^{(s)} X^{j}, \quad \lambda_{j}^{(s)} \in \mathbb{Q}[\pi]
$$

are given by

$$
\lambda_{0}^{(s)}=\frac{2^{2 s-1}(-1)^{s-1} B_{2 s}}{(2 s) !} \pi^{2 s}
$$

and (i) for $s=1,2$,

$$
\begin{aligned}
& \lambda_{1}^{(1)}=-2, \quad \lambda_{2}^{(1)}=\frac{2}{9} \pi^{2}+\frac{2}{3}, \quad \lambda_{3}^{(1)}=-\frac{8}{3}, \quad \lambda_{4}^{(1)}=\frac{16}{135} \pi^{2}+\frac{8}{9}, \\
& \lambda_{1}^{(2)}=0, \quad \lambda_{2}^{(2)}=\frac{4}{135} \pi^{4}-\frac{4}{9} \pi^{2}, \quad \lambda_{3}^{(2)}=\frac{16}{3},
\end{aligned}
$$

(ii) for $s \geq 3$,

$\lambda_{1}^{(s)}=0, \quad \lambda_{2}^{(s)}=\frac{2^{2 s-1}(-1)^{s-1}}{3 \cdot(2 s-1) !} \pi^{2 s-2}\left(2 B_{2 s} \pi^{2}+s(2 s-1) B_{2 s-2}\right), \quad \lambda_{3}^{(s)}=0$.

Theorem 2. For $-1<\beta<-1+\delta_{0}$, we have

$$
(\alpha+\beta)^{2 s-1} g_{2 s-1}^{*}=\Psi_{s}(\eta)\left(1+O\left(e^{-\pi^{2} /(2 \eta)}\right)\right) .
$$

Here $\delta_{0}$ and $\eta$ are as in Theorem 1 , and $\Psi_{s}(X)$ is an entire function written in the form

$$
\begin{aligned}
& \Psi_{s}(X):=\frac{\left(X^{-1} \sinh X\right)^{2 s-1}}{(2 s-2) !} \\
& \quad \times \sum_{p=0}^{s-1}(-1)^{p} 2^{-2 p-2} \pi^{2 p+1} \tau_{s-1-p}(s-1) E_{2 p} X^{2 s-2 p-2} .
\end{aligned}
$$


Several coefficients of the series expansion

$$
\Psi_{s}(X)=\sum_{j=0}^{\infty} \mu_{j}^{(s)} X^{2 j}, \quad \mu_{j}^{(s)} \in \mathbb{Q}[\pi]
$$

are given by

$$
\mu_{0}^{(s)}=\frac{(-1)^{s-1} E_{2 s-2}}{2^{2 s}(2 s-2) !} \pi^{2 s-1},
$$

and (i) for $s=1$,

$$
\mu_{m}^{(1)}=\frac{\pi}{4 \cdot(2 m+1) !} \quad(m \geq 1)
$$

(ii) for $s \geq 2$,

$$
\mu_{1}^{(s)}=\frac{(-1)^{s-1}(2 s-1)}{2^{2 s+1} \cdot 3 \cdot(2 s-2) !} \pi^{2 s-3}\left(E_{2 s-2} \pi^{2}+8(s-1)(2 s-3) E_{2 s-4}\right) .
$$

Remark 1. The asymptotic formulas above are also valid as $\beta \rightarrow-1$ through the sector $|\arg (\beta+1)|<\pi / 2-\theta_{0}$, where $\theta_{0}>0$ is an arbitrary small number (then $\eta \rightarrow 0,|\arg \eta|<\pi / 2-\theta_{0}^{\prime}$ for some $\theta_{0}^{\prime}>0$ ) (see Remark 4 and Section 5).

Remark 2. The quantity $\beta$ may be regarded as a function of the modulus $k$ of Jacobian elliptic functions, which satisfies $\beta \rightarrow-1+0$ as $k \rightarrow 1-0$ (see Section 4). Moreover, $\beta \rightarrow i$ and $\alpha \rightarrow i$ as $k \rightarrow \infty$ through a suitable sector. In this case, for

$$
(\alpha-\beta)^{2 s} h_{2 s}=\sum_{n=1}^{\infty} \frac{1}{U_{2 n}^{2 s}} \quad \text { and } \quad(\alpha-\beta)^{2 s-1} f_{2 s-1}=\sum_{n=1}^{\infty} \frac{1}{U_{2 n-1}^{2 s-1}},
$$

we obtain similar asymptotic formulas as $\beta \rightarrow i$.

\section{Euler's formulas for zeta functions}

For $-1<\beta<-1+\delta_{0}$ and for $n \geq 1$, observing that

$$
\begin{aligned}
\left|\frac{\alpha^{2 n}-\beta^{2 n}}{\alpha^{2}-\beta^{2}}\right| & =\left|\sum_{\nu=0}^{n-1} \alpha^{2 n-2-2 \nu} \beta^{2 \nu}\right| \\
& =\left|\sum_{\nu=0}^{n-1} \beta^{4 \nu-2 n+2}\right|=\left|\frac{1}{2} \sum_{\nu=0}^{n-1}\left(\beta^{4 \nu-2 n+2}+\beta^{-(4 \nu-2 n+2)}\right)\right| \geq n,
\end{aligned}
$$

we have for $s \in \mathbb{N}$

$$
\lim _{\beta \rightarrow-1+0}\left(\alpha^{2}-\beta^{2}\right)^{2 s} h_{2 s}=\sum_{n=1}^{\infty} \frac{1}{n^{2 s}}
$$


Therefore, letting $\beta$ tend to $-1+0$ in Theorem 1 , we obtain

$$
\zeta(2 s)=\frac{2^{2 s-1}(-1)^{s-1} B_{2 s}}{(2 s) !} \pi^{2 s} \quad(s \in \mathbb{N}) .
$$

For each $s \in \mathbb{N}$ a similar argument concerning Theorem 2 leads us

$$
\lim _{\beta \rightarrow-1+0}(\alpha+\beta)^{2 s-1} g_{2 s-1}^{*}=\sum_{n=1}^{\infty} \frac{(-1)^{n+1}}{(2 n-1)^{2 s-1}}=\frac{(-1)^{s-1} E_{2 s-2}}{2^{2 s}(2 s-2) !} \pi^{2 s-1} .
$$

\section{Preliminaries}

Consider the complete elliptic integrals of the first and the second kinds with the modulus $k$

(5)

$$
K=K(k):=\int_{0}^{1} \frac{d t}{\sqrt{\left(1-t^{2}\right)\left(1-k^{2} t^{2}\right)}}, \quad E=E(k):=\int_{0}^{1} \sqrt{\frac{1-k^{2} t^{2}}{1-t^{2}}} d t
$$

for $k^{2} \in \mathbb{C} \backslash(\{0\} \cup[1,+\infty))$, where the branch of each integrand is chosen so that it tends to 1 as $t \rightarrow 0$. Put

$$
\beta=\beta(k)=-e^{-\pi K^{\prime} /(2 K)}, \quad \alpha=\alpha(k)=-1 / \beta(k),
$$

where

$$
K^{\prime}:=K\left(k^{\prime}\right), \quad k^{\prime}:=\sqrt{1-k^{2}},
$$

the branch of $k^{\prime}$ being chosen so that $k^{\prime}>0$ for $0<k<1$. These integrals are expressible as follows (cf. $[1,17.3 .9$ and 17.3.10]):

$$
\begin{aligned}
K & =\frac{\pi}{2} F\left(1 / 2,1 / 2,1, k^{2}\right), \\
K^{\prime} & =\frac{\pi}{2} F\left(1 / 2,1 / 2,1,1-k^{2}\right), \\
E & =\frac{\pi}{2} F\left(-1 / 2,1 / 2,1, k^{2}\right),
\end{aligned}
$$

with the hypergeometric function

$$
F(a, b, c, x)=\sum_{n=0}^{\infty} \frac{(a)_{n}(b)_{n}}{(c)_{n} n !} x^{n}, \quad(a)_{n}:=\frac{\Gamma(a+n)}{\Gamma(a)}=a(a+1) \cdots(a+n-1) .
$$

Lemma 3. As $k \rightarrow 1-0$, we have

$$
\begin{aligned}
K & =l(k)\left(1+2 \log 2 \cdot l(k)^{-1}\right)\left(1+O\left(1-k^{2}\right)\right), \\
K^{\prime} & =\frac{\pi}{2}\left(1+O\left(1-k^{2}\right)\right), \\
E & =1+O\left(\left(1-k^{2}\right) l(k)\right),
\end{aligned}
$$

with

$$
l(k):=\log \left(1 / k^{\prime}\right)=\log \left(1 / \sqrt{1-k^{2}}\right) .
$$


Furthermore $l(k) \rightarrow+\infty$ and $K \rightarrow+\infty$ as $k \rightarrow 1-0$.

Proof. Formula (12) follows immediately from (9). Recall the connection formula for $F(1 / 2,1 / 2,1, z)$ around $z=0$ and $z=1$ :

$$
\begin{aligned}
& F(1 / 2,1 / 2,1, z)= \\
& \frac{\Gamma(1)}{\Gamma(1 / 2)^{2}} \sum_{n=0}^{\infty} \frac{(1 / 2)_{n}^{2}}{(n !)^{2}}(2 \psi(n+1)-2 \psi(n+1 / 2)-\log (1-z))(1-z)^{n}, \\
& \psi(t)=\frac{\Gamma^{\prime}(t)}{\Gamma(t)}=-\gamma+\sum_{m=0}^{\infty}\left(\frac{1}{m+1}-\frac{1}{m+t}\right)
\end{aligned}
$$

for $|1-z|<1,|\arg (1-z)|<\pi$ (cf. $[1,15.3 .10]$ ). Putting $z=k^{2}$, we derive for $\left|1-k^{2}\right|<1,\left|\arg \left(1-k^{2}\right)\right|<\pi$,

$$
\begin{aligned}
K= & \frac{1}{2} \sum_{n=0}^{\infty} \frac{(1 / 2)_{n}^{2}}{(n !)^{2}} \\
& \times\left(2 \sum_{m=0}^{\infty}\left(\frac{1}{m+n+1 / 2}-\frac{1}{m+n+1}\right)-\log \left(1-k^{2}\right)\right)\left(1-k^{2}\right)^{n} \\
= & 2 \log 2-\frac{1}{2} \log \left(1-k^{2}\right)+O\left(\left(1-k^{2}\right) \log \left(1-k^{2}\right)\right) \\
= & l(k)+2 \log 2+O\left(\left(1-k^{2}\right) l(k)\right),
\end{aligned}
$$

which implies (11), where the branch of $\log \left(1-k^{2}\right)$ is chosen so that $\operatorname{Im} \log \left(1-k^{2}\right)=0$ for $0<k<1$, namely for $\arg k=\arg (1-k)=0$. Using the formula

$$
\begin{aligned}
& \begin{array}{l}
F(-1 / 2,1 / 2,1, z)= \\
\quad \frac{\Gamma(1)^{2}}{\Gamma(1 / 2) \Gamma(3 / 2)}+\frac{\Gamma(1)}{\Gamma(-1 / 2) \Gamma(1 / 2)} \sum_{n=0}^{\infty} \frac{(1 / 2)_{n}(3 / 2)_{n}}{n !(n+1) !}(1-z)^{n+1} \\
\times(\log (1-z)-\psi(n+1)-\psi(n+2)+\psi(n+1 / 2)+\psi(n+3 / 2))
\end{array} \\
& \text { for }|1-z|<1,|\arg (1-z)|<\pi(\operatorname{cf}[1,15.3 .11]), \text { we obtain (13). }
\end{aligned}
$$

From (11) it follows that

$$
1-k^{2}=e^{-2 l(k)}=O\left(e^{-2 K}\right)
$$

as $k \rightarrow 1-0$. Hence, by (12), for $1-\delta_{1}<k<1$,

$$
\beta(k)=-\exp \left(-\frac{\pi^{2}}{4 K}\left(1+O\left(e^{-2 K}\right)\right)\right),
$$

and $|\beta(k)|<1$, provided that $\delta_{1}>0$ is sufficiently small. This implies that $\beta(k) \rightarrow-1+0$ as $k \rightarrow 1-0$. 
Remark 3. The connection formulas in the proof of Lemma 3 are also valid, as long as we consider the analytic continuation of $\log \left(1-k^{2}\right)$ on the universal covering of the punctured disk $0<|k-1|<1$. Hence as $k \rightarrow 1$ (without any condition on $\arg \left(1-k^{2}\right)$ ) we have $\operatorname{Re} K(k) \rightarrow+\infty$ and $\beta(k) \rightarrow-1,|\arg (\beta(k)+1)|<\pi / 2-\theta_{0}$, where $\theta_{0}$ is as in Remark 1. Conversely, for every path $C_{\beta}$ tending to $\beta=-1$ through the sector $|\arg (\beta+1)|<\pi / 2$, there exists a curve $C_{k}$ tending to $k=1$ such that $\beta\left(C_{k}\right)=C_{\beta}$.

Lemma 4. There exists a small positive number $\delta_{1}$ such that, for $1-\delta_{1}<$ $k<1$, the quantities $\beta=\beta(k)$ and $\alpha=\alpha(k)=-1 / \beta(k)$ satisfy

$$
\begin{aligned}
\alpha(k)^{2}-\beta(k)^{2} & =\left(e^{\pi^{2} /(2 K)}-e^{-\pi^{2} /(2 K)}\right)\left(1+O\left(e^{-2 K}\right)\right), \\
\alpha(k)+\beta(k) & =\left(e^{\pi^{2} /(4 K)}-e^{-\pi^{2} /(4 K)}\right)\left(1+O\left(e^{-2 K}\right)\right) .
\end{aligned}
$$

Proof. Using (6), (12) and (14), we have

$$
\begin{aligned}
\alpha(k)^{2}-\beta(k)^{2} & =e^{\pi K^{\prime} / K}-e^{-\pi K^{\prime} / K} \\
& =e^{-\pi K^{\prime} / K}\left(e^{2 \pi K^{\prime} / K}-1\right) \\
& =\exp \left(-\frac{\pi^{2}}{2 K}\left(1+O\left(e^{-2 K}\right)\right)\right)\left(\exp \left(\frac{\pi^{2}}{K}\left(1+O\left(e^{-2 K}\right)\right)\right)-1\right) \\
& =e^{\frac{-\pi^{2}}{2 K}}\left(1+O\left(K^{-1} e^{-2 K}\right)\right)\left(e^{\frac{\pi^{2}}{K}}\left(1+O\left(K^{-1} e^{-2 K}\right)\right)-1\right) \\
& =e^{-\pi^{2} /(2 K)}\left(1+O\left(e^{-2 K}\right)\right)\left(\left(e^{\pi^{2} / K}-1\right)+O\left(K^{-1} e^{-2 K}\right)\right) \\
& =e^{-\pi^{2} /(2 K)}\left(e^{\pi^{2} / K}-1\right)\left(1+O\left(e^{-2 K}\right)\right)
\end{aligned}
$$

as $k \rightarrow 1-0$ (and $K \rightarrow+\infty$ ), which implies (16). Estimate (17) is obtained by a similar argument.

We note the following lemmas concerning the Jacobian elliptic functions

$$
\operatorname{ns}(k, z)=1 / \operatorname{sn}(k, z), \quad \operatorname{cn}(k, z)=\sqrt{1-\operatorname{sn}^{2}(k, z)}
$$

with $w=\operatorname{sn}(k, z)$ defined by

$$
z=\int_{0}^{w} \frac{d v}{\sqrt{\left(1-v^{2}\right)\left(1-k^{2} v^{2}\right)}} .
$$

Lemma 5. Around $z=0$,

$$
\mathrm{ns}^{2}(k, z)=z^{-2}+\sum_{j=0}^{\infty} c_{j}(k) z^{2 j}
$$

where $c_{j}(k) \in \mathbb{Q}\left[k^{2}\right]$. In particular,

$$
\mathrm{ns}^{2}(1, z)=\operatorname{coth}^{2} z=z^{-2}+\sum_{j=0}^{\infty} c_{j}(1) z^{2 j},
$$


where

$$
c_{0}(1)=\frac{2}{3}, \quad c_{j}(1)=-\frac{2^{2 j+2}(2 j+1) B_{2 j+2}}{(2 j+2) !} \quad(j \geq 1) .
$$

Proof. The function $u=\mathrm{ns}^{2}(k, z)$ satisfies the differential equations

$$
\left(u^{\prime}\right)^{2}=4 u(u-1)\left(u-k^{2}\right) \text { and } u^{\prime \prime}=6 u^{2}-4\left(k^{2}+1\right) u+2 k^{2} .
$$

Substituting the expansion $z^{-2}+\sum_{j=0}^{\infty} c_{j}(k) z^{2 j}$ of $\mathrm{ns}^{2}(k, z)$ into these equations, we can determine the coefficients $c_{j}(k)$ (see [4, Lemma 2] and the recursive relation in it). The relation $\mathrm{ns}^{2}(1, z)=\operatorname{coth}^{2} z$ immediately follows from the fact that $u=\mathrm{ns}^{2}(1, z)$ satisfies the equation $\left(u^{\prime}\right)^{2}=4 u(u-1)^{2}$ admitting the general solution $\operatorname{coth}^{2}\left(z-z_{0}\right)$.

The function $u=k \operatorname{cn}(k, z)$ satisfies

$$
\left(u^{\prime}\right)^{2}=\left(k^{2}-u^{2}\right)\left(u^{2}+1-k^{2}\right), \quad u(0)=k .
$$

If $k=1$, then $\left(u^{\prime}\right)^{2}=u^{2}\left(1-u^{2}\right)$ admits the general solution $\operatorname{sech}\left(z-z_{0}\right)$. From this fact we obtain the following (see also [6, Lemma 2]):

Lemma 6. Around $z=0$,

$$
k \operatorname{cn}(k, z)=k+\sum_{j=1}^{\infty} c_{j}^{*}(k) z^{2 j}
$$

where $c_{j}^{*}(k) \in \mathbb{Q}[k]$. In particular,

$$
\operatorname{cn}(1, z)=\operatorname{sech} z=1+\sum_{j=1}^{\infty} c_{j}^{*}(1) z^{2 j}, \quad c_{j}^{*}(1)=\frac{E_{2 j}}{(2 j) !} .
$$

\section{Proofs of results}

5.1. Proof of Theorem 1. Recall that

$$
\begin{aligned}
\left(\alpha^{2}-\beta^{2}\right)^{2 s} h_{2 s} & =\left(\frac{\alpha^{2}-\beta^{2}}{2}\right)^{2 s} \sum_{\nu=1}^{\infty} \operatorname{cosech}^{2 s}\left(\nu \pi K^{\prime} / K\right) \\
& =\frac{\left(\alpha^{2}-\beta^{2}\right)^{2 s}}{(2 s-1) !} \sum_{j=0}^{s-1} \sigma_{s-j-1}(s) A_{2 j+1}\left(\beta^{2}\right),
\end{aligned}
$$

where

$$
A_{2 j+1}(q):=\sum_{n=1}^{\infty} \frac{n^{2 j+1} q^{2 n}}{1-q^{2 n}}
$$

(cf. [12, Table $1(\mathrm{i})],[4])$. The series $A_{2 j+1}\left(\beta(k)^{2}\right)$ are generated by

$$
\left(\frac{2 K}{\pi}\right)^{2} \mathrm{~ns}^{2}\left(\frac{2 K x}{\pi}\right)=\frac{4 K(K-E)}{\pi^{2}}+\operatorname{cosec}^{2} x-8 \sum_{j=0}^{\infty}(-1)^{j} A_{2 j+1} \frac{(2 x)^{2 j}}{(2 j) !}
$$


(cf. [12, Tables 1(i)], [9], [11, p. 535]), namely

$$
\begin{aligned}
& A_{1}=\frac{1}{24}\left(1-\left(\frac{2 K}{\pi}\right)^{2}\left(\frac{3 E}{K}-2+k^{2}\right)\right) \\
& \left(\frac{2 K}{\pi}\right)^{2 j+2} c_{j}(k)=a_{j}-(-1)^{j} \frac{2^{2 j+3}}{(2 j) !} A_{2 j+1} \quad(j \geq 1) .
\end{aligned}
$$

Here $c_{j}(k)$ are the coefficients given in Lemma 5 , and $a_{j}$ are the coefficients of the expansion

$$
\operatorname{cosec}^{2} z=z^{-2}+\sum_{j=0}^{\infty} a_{j} z^{2 j}
$$

namely, $a_{0}=1 / 3, a_{j}=(-1)^{j-1} c_{j}(1)(j \geq 1)$.

Recall that $K \rightarrow+\infty$ and that $\beta(k) \rightarrow-1+0$ as $k \rightarrow 1-0$ (cf. (15) and Lemma 3). By (16) we have

$$
\begin{aligned}
\left(\alpha^{2}-\beta^{2}\right)^{2 s} & =\left(e^{\pi^{2} / K}+e^{-\pi^{2} / K}-2\right)^{s}\left(1+O\left(e^{-2 K}\right)\right) \\
& =\pi^{4 s} K^{-2 s}\left(2 \pi^{-4} K^{2}\left(\cosh \left(\pi^{2} / K\right)-1\right)\right)^{s}\left(1+O\left(e^{-2 K}\right)\right)
\end{aligned}
$$

as $k \rightarrow 1-0$. Note that

$$
2 \pi^{-4} K^{2}\left(\cosh \left(\pi^{2} / K\right)-1\right)=\sum_{m=1}^{\infty} \frac{2 \pi^{4(m-1)}}{(2 m) !} K^{-2(m-1)}=1+O\left(K^{-2}\right) .
$$

By (19), (14) and Lemma 3 , the quantity $A_{1}\left(\beta^{2}\right)$ can be written as

$$
\begin{aligned}
A_{1} & =\frac{1}{24}\left(1-\frac{4 K^{2}}{\pi^{2}}\left(\frac{3}{K}\left(1+O\left(l(k) e^{-2 K}\right)\right)-1+O\left(e^{-2 K}\right)\right)\right) \\
& =\frac{1}{24}\left(1-\frac{4 K^{2}}{\pi^{2}}\left(-1+3 K^{-1}+O\left(e^{-2 K}\right)\right)\right) \\
& =\frac{K^{2}}{6 \pi^{2}}\left(1-3 K^{-1}+\frac{\pi^{2}}{4} K^{-2}\right)\left(1+O\left(e^{-2 K}\right)\right) .
\end{aligned}
$$

Combining this with (21), we have for $s \geq 1$

$$
\begin{aligned}
\left(\alpha^{2}-\beta^{2}\right)^{2 s} A_{1}=\frac{\pi^{4 s-2}}{6} & K^{-2(s-1)}\left(2 \pi^{-4} K^{2}\left(\cosh \left(\pi^{2} / K\right)-1\right)\right)^{s} \\
\times & \left(1-3 K^{-1}+\frac{\pi^{2}}{4} K^{-2}\right)\left(1+O\left(e^{-2 K}\right)\right)
\end{aligned}
$$


as $k \rightarrow 1-0$. For $s \geq 2$ and for $2 \leq p \leq s$, using (20), (21), and Lemma 5 with the facts $a_{p-1}=(-1)^{p} c_{p-1}(1), c_{p-1}(k)=c_{p-1}(1)+O\left(e^{-2 K}\right)$, we have

$$
\begin{aligned}
\left(\alpha^{2}-\beta^{2}\right)^{2 s} A_{2 p-1}= & \frac{(-1)^{p}(2 p-2) !}{2} \pi^{4 s-2 p} c_{p-1}(1) K^{-2(s-p)} \\
& \times\left(2 \pi^{-4} K^{2}\left(\cosh \left(\pi^{2} / K\right)-1\right)\right)^{s} \\
& \times\left(1-(-1)^{p} 2^{-2 p} \pi^{2 p} K^{-2 p}\right)\left(1+O\left(e^{-2 K}\right)\right) \\
= & \frac{(-1)^{p-1}}{p} 2^{2 p-2} \pi^{4 s-2 p} B_{2 p} K^{-2(s-p)} \\
& \times\left(2 \pi^{-4} K^{2}\left(\cosh \left(\pi^{2} / K\right)-1\right)\right)^{s} \\
& \times\left(1-(-1)^{p} 2^{-2 p} \pi^{2 p} K^{-2 p}\right)\left(1+O\left(e^{-2 K}\right)\right)
\end{aligned}
$$

as $k \rightarrow 1-0$. By (15),

$$
\begin{aligned}
& K^{-1}=-\frac{4}{\pi^{2}} \log (-\beta)\left(1+O\left(e^{-2 K}\right)\right), \\
& K=\frac{\pi^{2}}{4 \eta}\left(1+O\left(e^{-2 K}\right)\right)=\frac{\pi^{2}}{4 \eta}+O\left(K e^{-2 K}\right)=\frac{\pi^{2}}{4 \eta}+O(1),
\end{aligned}
$$

and hence

$$
K^{-1}=\frac{4}{\pi^{2}} \eta\left(1+O\left(e^{-\pi^{2} /(2 \eta)}\right)\right), \quad e^{-2 K}=O\left(e^{-\pi^{2} /(2 \eta)}\right) .
$$

For $s \geq 1$ we derive from (18), (22) and (23) that

$$
\begin{aligned}
\left(\alpha^{2}-\beta^{2}\right)^{2 s} h_{2 s} & =\frac{\left(\alpha^{2}-\beta^{2}\right)^{2 s}}{(2 s-1) !} \sum_{p=1}^{s} \sigma_{s-p}(s) A_{2 p-1} \\
& =\frac{\left(2 \pi^{-4} K^{2}\left(\cosh \left(\pi^{2} / K\right)-1\right)\right)^{s}}{(2 s-1) !} \sum_{p=1}^{s} \sigma_{s-p}(s) F_{s, p}(K)
\end{aligned}
$$

with

$$
F_{s, 1}(K):=\frac{\pi^{4 s-2}}{6} K^{-2(s-1)}\left(1-3 K^{-1}+\frac{\pi^{2}}{4} K^{-2}\right)\left(1+O\left(e^{-2 K}\right)\right),
$$

and

$$
\begin{aligned}
F_{s, p}(K):=\frac{(-1)^{p-1}}{p} 2^{2 p-2} & \pi^{4 s-2 p} B_{2 p} K^{-2(s-p)} \\
& \times\left(1-(-1)^{p} 2^{-2 p} \pi^{2 p} K^{-2 p}\right)\left(1+O\left(e^{-2 K}\right)\right)
\end{aligned}
$$

for $p \geq 2$. Now we note the following: for a function $\phi(z)$ holomorphic for $|z|<r_{0}$ satisfying $\phi(0) \neq 0$,

$$
\phi(z+\Delta z)=\phi(z)+\int_{0}^{\Delta z} \phi^{\prime}(z+t) d t=\phi(z)+O(\Delta z)=\phi(z)(1+O(\Delta z))
$$


as $\Delta z \rightarrow 0$, uniformly for $|z|<r_{0}^{\prime}$, provided that $r_{0}^{\prime}$ is sufficiently small. Substituting (24) into (25), and using this fact with $z=4 \pi^{-2} \eta$ and $\Delta z=$ $O\left(\eta e^{-\pi^{2} /(2 \eta)}\right)$, we have the required formula. Using

$$
2 X^{-2}(\cosh (4 X)-1)=\sum_{m=0}^{\infty} \frac{2^{4 m+5}}{(2 m+2) !} X^{2 m}
$$

and

$$
\sigma_{1}(s)=-\sum_{r=1}^{s-1} r^{2}=-\frac{1}{6} s(s-1)(2 s-1),
$$

we obtain the coefficients mentioned in the theorem.

5.2. Proof of Theorem 2. Note that, for $s \in \mathbb{N}$,

$$
\begin{aligned}
(\alpha+\beta)^{2 s-1} g_{2 s-1}^{*} & =\left(\frac{\alpha+\beta}{2}\right)^{2 s-1} \sum_{\nu=1}^{\infty}(-1)^{\nu+1} \operatorname{cosech}^{2 s-1}\left((\nu-1 / 2) \pi K^{\prime} / K\right) \\
(26) \quad & =\frac{(\alpha+\beta)^{2 s-1}}{2^{2 s-2}(2 s-2) !} \sum_{j=0}^{s-1} \tau_{s-1-j}(s-1) T_{2 j}\left(\beta^{2}\right),
\end{aligned}
$$

where

$$
T_{2 j}(q):=\sum_{n=1}^{\infty} \frac{(2 n-1)^{2 j} q^{n-1 / 2}}{1+q^{2 n-1}}
$$

(cf. [12, Table $1(\mathrm{xv})]$ ). The series $T_{2 j}\left(\beta(k)^{2}\right)$ are generated by

$$
\left(\frac{2 K}{\pi}\right) k \operatorname{cn}\left(\frac{2 K x}{\pi}\right)=4 \sum_{j=0}^{\infty}(-1)^{j} T_{2 j} \frac{x^{2 j}}{(2 j) !}
$$

namely

$$
\left(\frac{2 K}{\pi}\right)^{2 j+1} c_{j}^{*}(k)=\frac{(-1)^{j} 4}{(2 j) !} T_{2 j} \quad(j \geq 0),
$$

where $c_{j}^{*}(k)$ are coefficients given in Lemma 6. By (17)

$$
\begin{aligned}
\alpha+\beta & =\left(e^{\pi^{2} /(4 K)}-e^{-\pi^{2} /(4 K)}\right)\left(1+O\left(e^{-2 K}\right)\right) \\
& =\frac{\pi^{2}}{2} K^{-1}\left(4 \pi^{-2} K \sinh \left(\pi^{2} /(4 K)\right)\right)\left(1+O\left(e^{-2 K}\right)\right),
\end{aligned}
$$

where

$$
4 \pi^{-2} K \sinh \left(\pi^{2} /(4 K)\right)=\sum_{m=1}^{\infty} \frac{\pi^{4(m-1)}}{4^{2(m-1)}(2 m-1) !} K^{-2(m-1)}=1+O\left(K^{-2}\right) .
$$

Note that $c_{j-1}^{*}(k)=c_{j-1}^{*}(1)+O(1-k)=c_{j-1}^{*}(1)+O\left(e^{-2 K}\right)$ as $k \rightarrow 1-0$, because $c_{j-1}^{*}(k) \in \mathbb{Q}[k]$. By $(27),(28)$ and Lemma 6 , we have, for $s \geq 1$ 
and for $0 \leq p \leq s-1$,

$$
\begin{aligned}
(\alpha+\beta)^{2 s-1} T_{2 p}=( & -1)^{p} 2^{2 p-2 s} \pi^{4 s-2 p-3} E_{2 p} K^{-2(s-p-1)} \\
& \times\left(4 \pi^{-2} K \sinh \left(\pi^{2} /(4 K)\right)\right)^{2 s-1}\left(1+O\left(e^{-2 K}\right)\right) .
\end{aligned}
$$

Substituting these quantities into (26), we obtain

$$
\begin{aligned}
(\alpha+\beta)^{2 s-1} g_{2 s-1}^{*}= & \frac{(\alpha+\beta)^{2 s-1}}{2^{2 s-2}(2 s-2) !} \sum_{p=0}^{s-1} \tau_{s-1-p}(s-1) T_{2 p} \\
= & \frac{1}{(2 s-2) !}\left(4 \pi^{-2} K \sinh \left(\pi^{2} /(4 K)\right)\right)^{2 s-1} \\
& \quad \times \sum_{p=0}^{s-1}\left((-1)^{p} 2^{2 p-4 s+2} \pi^{4 s-2 p-3} \tau_{s-1-p}(s-1)\right. \\
& \left.\quad \times E_{2 p} K^{-2(s-p-1)}\left(1+O\left(e^{-2 K}\right)\right)\right) .
\end{aligned}
$$

This combined with (24) yields the required formula. Using

$$
2 X^{-1} \sinh X=2 \sum_{m=0}^{\infty} \frac{X^{2 m}}{(2 m+1) !}
$$

and

$$
\tau_{1}(s-1)=-\sum_{r=1}^{s-1}(2 r-1)^{2}=-\frac{1}{3}(s-1)(2 s-1)(2 s-3),
$$

we can check the coefficients given in the theorem.

\section{Acknowledgements}

The authors are grateful to Professor Yu. V. Nesterenko for stimulating discussions on the degeneration of reciprocal sums of Fibonacci numbers, and also to the referee for his/her very helpful comments.

\section{References}

[1] M. Abramowitz and I. A. Stegun, Handbook of Mathematical Functions. Dover, New York, 1965.

[2] D. Duverney, Ke. Nishioka, Ku. Nishioka, and I. Shiokawa, Transcendence of Jacobi's theta series. Proc. Japan Acad. Ser. A Math. Sci. 72 (1996), 202-203.

[3] D. Duverney, Ke. Nishioka, Ku. Nishioka, And I. Shiokawa, Transcendence of RogersRamanujan continued fraction and reciprocal sums of Fibonacci numbers. Proc. Japan Acad. Ser. A Math. Sci. 73 (1997), 140-142.

[4] C. Elsner, S. Shimomura, And I. Shiokawa, Algebraic relations for reciprocal sums of Fibonacci numbers. Acta Arith. 130 (2007), 37-60.

[5] C. Elsner, S. Shimomura, and I. Shiokawa, Algebraic relations for reciprocal sums of odd terms in Fibonacci numbers. Ramanujan J. 17 (2008), 429-446.

[6] C. Elsner, S. Shimomura, And I. Shiokawa, Algebraic relations for reciprocal sums of even terms in Fibonacci numbers. To appear in St. Petersburg Math. J.

[7] H. Hancock, Theory of Elliptic Functions. Dover, New York, 1958. 
[8] A. Hurwitz And R. Courant, Vorlesungen über allgemeine Funktionentheorie und elliptische Funktionen. Springer, Berlin, 1925.

[9] C. G. J. JACOBI, Fundamenta Nova Theoriae Functionum Ellipticarum. Königsberg, 1829.

[10] Yu. V. Nesterenko, Modular functions and transcendence questions. Mat. Sb. 187 (1996), 65-96; English transl. Sb. Math. 187 (1996), 1319-1348.

[11] E. T. Whittaker and G. N. Watson, Modern Analysis, 4th ed. Cambridge Univ. Press, Cambridge, 1927.

[12] I. J. ZUCKER, The summation of series of hyperbolic functions. SIAM J. Math. Anal. 10 (1979), 192-206.

Carsten ELSNER

Fachhochschule für die Wirtschaft

University of Applied Sciences

Freundallee 15

30173 Hannover, Germany

E-mail: carsten.elsner@fhdw.de

Shun Shimomura

Department of Mathematics

Keio University

3-14-1 Hiyoshi, Kohoku-ku

Yokohama 223-8522 Japan

E-mail: shimomur@math.keio.ac.jp

Iekata SHIOKAWA

Department of Mathematics

Keio University

3-14-1 Hiyoshi, Kohoku-ku

Yokohama 223-8522 Japan

E-mail: shiokawa@beige.ocn.ne.jp 